\title{
Long-acting muscarinic antagonists (LAMA) added to inhaled corticosteroids (ICS) versus higher dose ICS for adults with asthma (Protocol)
}

Kew KM, Allison DE, Evans DJW, Boyter AC

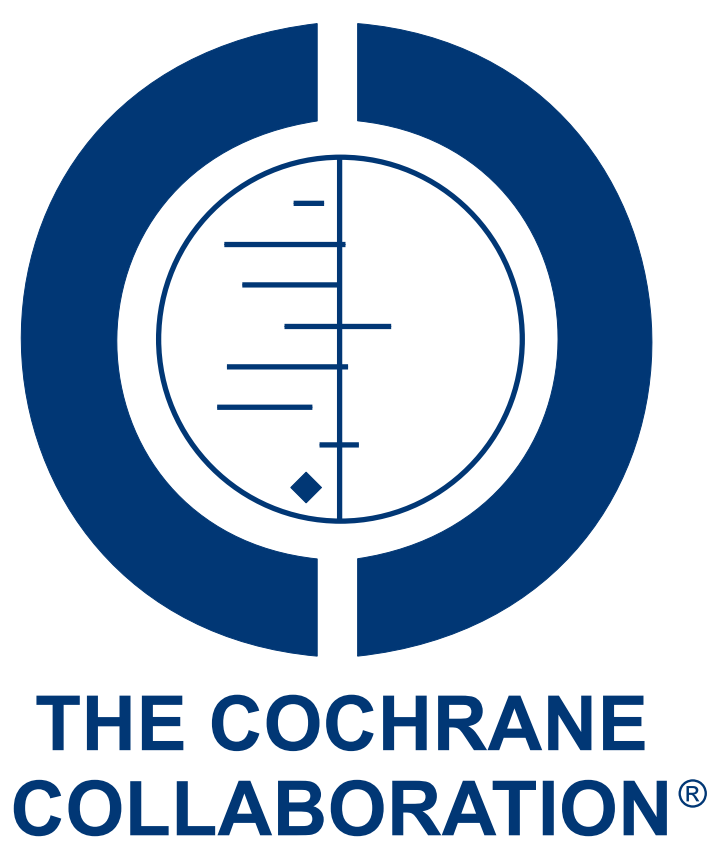

This is a reprint of a Cochrane protocol, prepared and maintained by The Cochrane Collaboration and published in The Cochrane Library 2014, Issue 12

http://www.thecochranelibrary.com

\section{WILEY}

Long-acting muscarinic antagonists (LAMA) added to inhaled corticosteroids (ICS) versus higher dose ICS for adults with asthma (Protocol)

Copyright $\odot 2014$ The Cochrane Collaboration. Published by John Wiley \& Sons, Ltd. 
TABLE OF CONTENTS

HEADER . . . . . . . . . . . . . . . . . . . . . . . . . . . . . . . . . . . . . . . 1

ABSTRACT . . . . . . . . . . . . . . . . . . . . . . . . . . . . . . . . . . . . . . . . . . . . .

BACKGROUND . . . . . . . . . . . . . . . . . . . . . . . . . . . . . . . . . . . . .

OBJECTIVES . . . . . . . . . . . . . . . . . . . . . . . . . . . . . . . . . . . . . . .

METHODS . . . . . . . . . . . . . . . . . . . . . . . . . . . . . . . . . . . . . .

ACKNOWLEDGEMENTS . . . . . . . . . . . . . . . . . . . . . . . . . . . . . . . . . . . . . . . .

REFERENCES . . . . . . . . . . . . . . . . . . . . . . . . . . . . . . . . . . . . . . 6

APPENDICES . . . . . . . . . . . . . . . . . . . . . . . . . . . . . . . . . . . . . . . 7

CONTRIBUTIONS OF AUTHORS . . . . . . . . . . . . . . . . . . . . . . . . . . . . . . . . . . . . . . . . . . . . .

DECLARATIONS OF INTEREST . . . . . . . . . . . . . . . . . . . . . . . . . . . . . . . . . . . 9

SOURCES OF SUPPORT . . . . . . . . . . . . . . . . . . . . . . . . . . . . . . . . . . .

Long-acting muscarinic antagonists (LAMA) added to inhaled corticosteroids (ICS) versus higher dose ICS for adults with asthma 
[Intervention Protocol]

\title{
Long-acting muscarinic antagonists (LAMA) added to inhaled corticosteroids (ICS) versus higher dose ICS for adults with asthma
}

\author{
Kayleigh M Kew ${ }^{1}$, Debbie E Allison ${ }^{2}$, David JW Evans ${ }^{3}$, Anne C Boyter ${ }^{2}$ \\ ${ }^{1}$ Population Health Research Institute, St George's, University of London, London, UK. ${ }^{2}$ Strathclyde Institute of Pharmacy and \\ Biomedical Sciences, University of Strathclyde, Glasgow, UK. ${ }^{3}$ Lancaster Patient Safety Research Unit, Royal Lancaster Infirmary, \\ Lancaster, UK
}

Contact address: Kayleigh M Kew, Population Health Research Institute, St George's, University of London, Cranmer Terrace, London, SW17 0RE, UK. kkew@sgul.ac.uk.

Editorial group: Cochrane Airways Group.

Publication status and date: New, published in Issue 12, 2014.

Citation: Kew KM, Allison DE, Evans DJW, Boyter AC. Long-acting muscarinic antagonists (LAMA) added to inhaled corticosteroids (ICS) versus higher dose ICS for adults with asthma. Cochrane Database of Systematic Reviews 2014, Issue 12. Art. No.: CD011437. DOI: 10.1002/14651858.CD011437.

Copyright (C) 2014 The Cochrane Collaboration. Published by John Wiley \& Sons, Ltd.

\begin{abstract}
A B S T R A C T
This is the protocol for a review and there is no abstract. The objectives are as follows:

To assess the efficacy and safety of adding a long-acting muscarinic agonist (LAMA) to any dose of inhaled corticosteroids (ICS) compared with increasing the dose of ICS for adults whose asthma is not well controlled.
\end{abstract}

\section{B A C K G R O U N D}

\section{Description of the condition}

Asthma is a 'common and potentially serious chronic disease' of the airways, which causes difficulty breathing due to narrowing of the airways, thickening of the airway walls and increased mucus production (GINA 2014). Asthma is recognised as a heterogeneous disease, but commonly causes symptoms including 'wheezing, shortness of breath, chest tightness and cough that vary over time in their occurrence, frequency and intensity' (GINA 2014). Around the world and particularly in low- and middle-income countries, asthma is frequently undiagnosed and untreated (Global Asthma Report 2011), and remains a significant cause of avoidable morbidity and mortality in developed countries such as the
UK (NRAD 2014), imposing 'a substantial burden on patients, their family and the community' (GINA 2014). Recent World Health Organisation estimates suggest 300 million people are affected worldwide, with direct treatment costs and indirect costs of lost productivity among the highest for non-communicable diseases (Global Asthma Report 2011). Prevalence estimates vary, and changes over time have been linked to various factors including air pollution, tobacco legislation, diet, and prevalence of other atopic diseases (Anderson 2005).

The two broad aims of asthma treatment are to maintain daily symptom control and prevent acute worsening of symptoms known as asthma attacks or 'exacerbations'. To achieve this, medication, usually given via an inhaler, is started at the most appropriate level based on severity and frequency of symptoms according to treatment 'steps' laid out in guidelines (e.g. GINA 2014). 
Depending on symptom control and frequency of exacerbations when treatment has been commenced, therapy can be 'stepped up' by increasing dose or adding medications to recapture control, or 'stepped down' to maintain patients at the lowest effective therapy and minimise side-effects.

\section{Description of the intervention}

The lowest treatment step in most guidelines is the sole use of a short-acting bronchodilating inhaler on an as-needed basis (e.g. salbutamol), which is often sufficient to treat mild or intermittent asthma symptoms. Regular use of low dose inhaled corticosteroids is the primary recommended preventer therapy for people with persistent asthma who remain inadequately controlled on asneeded medication alone (Step 2, GINA 2014). Regular ICS has been shown to improve lung function and reduce the need for reliever medications (Adams 2008; Adams 2008a). However, some people with asthma will continue to have symptoms and asthma attacks on ICS alone and guidelines suggest a range of treatment options for this group of patients (step 3 and above). Long-acting beta 2 -agonists (LABA) such as formoterol and salmeterol are the current preferred add-on therapy at step 3 (Ducharme 2008; GINA 2014) as they have been shown to have often small but statistically significant benefits on a range of outcomes over other treatment options such as increasing ICS dose (Ducharme 2010), adding theophylline (Tee 2009), or adding a leukotriene receptor antagonist (Chauhan 2014). Add-on drugs that allow ICS dose to be kept low are often seen as preferable since prolonged use of higher doses of ICS carries the risk of serious unwanted effects including growth retardation in children, decreased bone density, eye disorders, sleep problems, and anxiety (NICE 2013).

Long-acting muscarinic antagonists (LAMA), a class of drugs with proven effectiveness in COPD (Karner 2014) are now being considered as another add-on therapy for adults with asthma requiring more than ICS alone. Tiotropium, the first LAMA to be licensed in COPD and the most widely used, has demonstrated added benefits over LABA in terms of the frequency of exacerbations and hospital admissions for COPD, but not in terms of mortality or overall hospital admissions (Chong 2012). Evidence for the safety and efficacy of aclidinium bromide and glycopyrronium bromide, two LAMA formulations that have recently been licensed for use in COPD, is emerging but less well established (Ni 2014).

\section{How the intervention might work}

Long-acting muscarinic antagonists block receptors of the neurotransmitter acetylcholine on airway smooth muscle, glands and nerves, preventing muscle contraction and mucus secretion (Moulton 2011). The action on these receptors helps to alleviate symptoms of breathlessness, coughing and wheezing that characterise asthma (Lipworth 2014). These characteristics of LAMA and the overlap in pathophysiology and symptoms of asthma and COPD (Gosens 2006) have led to their testing in asthma as an add-on therapy for patients who do not achieve adequate control from standard-dose ICS alone, thus avoiding prolonged exposure to higher doses of ICS.

The most commonly reported side effect of LAMA for airways disease is dry mouth, with others including constipation or diarrhoea, cough, and headache (BNF). All LAMA for maintenance of airways disease are delivered via inhalers, either by powder (HandiHaler, Genuair, Breezhaler) or soft mist delivery (Respimat), and are not suitable to be used as rescue medication.

In COPD, there is conflicting evidence regarding the safety of tiotropium delivered via the Respimat device, with one recent observational study finding it increased the risk of death, particularly cardiac, compared with placebo via the HandiHaler device (Verhamme 2013). Another large randomised trial including over 17,000 people with COPD found no significant differences in long-term safety between the two devices (Wise 2013). As yet it is unclear whether differential safety profiles will be seen in people with asthma.

\section{Why it is important to do this review}

Only one preparation of LAMA (Spiriva Respimat $2.5 \mathrm{mcg}$ ) has been granted a UK license for use in severe asthma alongside LABA and ICS (eMC 2014). Following their demonstrated efficacy in COPD (Karner 2014), clinical trials are emerging to test the use of various LAMA regimens against the existing treatment options for asthma. One study found that nearly 30 per cent of patients who were uncontrolled on fluticasone remained so with the guideline recommended addition of LABA (Bateman 2004), suggesting there is a need for additional options for add-on therapy.

It is important to assess the safety of using LAMA add-on to ICS as an alternative to prolonged use of high doses of ICS that can have well established and undesirable side effects (NICE 2013). Alongside three other reviews also in production, this review will summarise the evidence to guide the possible use of LAMA addon as an alternative steroid-sparing agent. The other reviews will assess 1) LAMA add-on compared with LABA add-on 2) LAMA add-on compared with no change to ICS dose, and 3) LAMA addon as triple therapy with LABA+ICS compared with LABA+ICS alone.

\section{O B J E C T IVES}

To assess the efficacy and safety of adding a long-acting muscarinic agonist (LAMA) to any dose of inhaled corticosteroids (ICS) compared with increasing the dose of ICS for adults whose asthma is not well controlled. 


\section{METHODS}

\section{Criteria for considering studies for this review}

\section{Types of studies}

We will include double-blinded parallel or crossover randomised controlled trials (RCTs) of at least 12 weeks' duration. We will include studies reported as full-text, those published as abstract only, and unpublished data. The longer-term effects of ICS may not wash out between treatments in crossover trials, so we will perform a sensitivity analysis excluding them from the primary analyses.

We will not exclude studies on the basis of blinding.

\section{Types of participants}

We will include adults (18+) whose asthma is not well controlled on ICS alone. We will exclude trials that include participants with other chronic respiratory co-morbidities (e.g. chronic obstructive pulmonary disease, bronchiectasis).

If studies include adults and adolescents or children under 12 and data are not reported separately, we will include them if the mean age in both groups is over 18 .

\section{Types of interventions}

We will include studies randomising participants to receive any dose of the following LAMA preparations as an add-on to any dose of ICS:

- Tiotropium (Spiriva Handihaler or Respimat)

- Aclidinium bromide (Eklira Genuair)

- Glycopyrronium bromide (Seebri Breezhaler)

Eligible comparison group participants will be randomised to receive an increase in ICS dose.

We will include studies that permit the use of short-acting medications, e.g. salbutamol, terbutaline and ipratropium, as reliever therapy. However, to assess the effect of LAMA on top of ICS alone, we will exclude trials where:

- a long-acting beta 2 -agonist (LABA) was given as part of the randomised treatment (i.e. LAMA+ICS+LABA vs. ICS+LABA)

- participants were required to be taking a LABA to be included in the trial

- the majority of participants continued treatment with LABA alongside the randomised treatment

\section{Types of outcome measures}

1. Exacerbations requiring oral corticosteroids

2. Quality of life (measured on a validated asthma scale, e.g. Asthma Quality of Life Questionnaire)

3. Any serious adverse events

\section{Secondary outcomes}

1. Exacerbations requiring hospitalisation

2. Lung function (in particular, trough FEV1)

3. Asthma Control (measured on a validated scale, e.g. Asthma Control Questionnaire or Asthma Control Test)

4. Any adverse events

Reporting one or more of the outcomes listed here in the trial is not an inclusion criterion for the review.

If exacerbations are reported as a composite of more than one definition (e.g. patients with one of more exacerbation requiring hospitalisation or ED visit), we will analyse these separately.

\section{Search methods for identification of studies}

\section{Electronic searches}

We will identify trials from the Cochrane Airways Group's Specialised Register (CAGR), which is maintained by the Trials Search Co-ordinator for the Group. The Register contains trial reports identified through systematic searches of bibliographic databases including the Cochrane Central Register of Controlled Trials (CENTRAL), MEDLINE, EMBASE, CINAHL, AMED, and PsycINFO, and handsearching of respiratory journals and meeting abstracts (please see Appendix 1 for further details). We will search all records in the CAGR using the search strategy in Appendix 2. We will also conduct a search of ClinicalTrials.gov (www.ClinicalTrials.gov) and the WHO trials portal (www.who.int/ictrp/en/). We will search all databases from their inception to the present, and we will impose no restriction on language of publication.

\section{Searching other resources}

We will check reference lists of all primary studies and review articles for additional references. We will search relevant manufacturers' websites for trial information.

We will search for errata or retractions from included studies published in full-text on PubMed (www.ncbi.nlm.nih.gov/pubmed) and report the date this was done within the review.

\section{Data collection and analysis}


Two review authors (KK and DE) will independently screen titles and abstracts for inclusion of all the potential studies we identify as a result of the search and code them as 'retrieve' (eligible or potentially eligible/unclear) or 'do not retrieve'. We will retrieve the full-text study reports/publication and two review authors will independently screen the full-text and identify studies for inclusion, and identify and record reasons for exclusion of the ineligible studies. We will resolve any disagreement through discussion or, if required, we will consult a third person (DA or $\mathrm{AB}$ ). We will identify and exclude duplicates and collate multiple reports of the same study so that each study rather than each report is the unit of interest in the review. We will record the selection process in sufficient detail to complete a PRISMA flow diagram and 'Characteristics of excluded studies' table.

\section{Data extraction and management}

We will use a data collection form for study characteristics and outcome data which has been piloted on at least one study in the review. One review author (KK) will extract study characteristics from included studies. We will extract the following study characteristics.

1. Methods: study design, total duration of study, details of any 'run in' period, number of study centres and location, study setting, withdrawals, and date of study.

2. Participants: $N$, mean age, age range, gender, severity of condition, diagnostic criteria, baseline lung function, smoking history, inclusion criteria, and exclusion criteria.

3. Interventions: intervention, comparison, concomitant medications, and excluded medications.

4. Outcomes: primary and secondary outcomes specified and collected, and time points reported.

5. Notes: funding for trial, and notable conflicts of interest of trial authors.

Two review authors (KK and DE) will independently extract outcome data from included studies. We will note in the 'Characteristics of included studies' table if outcome data was not reported in a usable way. We will resolve disagreements by consensus or by involving a third person (DA or $\mathrm{AB}$ ). One review author (KK) will transfer data into the Review Manager (RevMan) file. We will double-check that data is entered correctly by comparing the data presented in the systematic review with the study reports. A second review author (DE) will spot-check study characteristics for accuracy against the trial reports.

\section{Assessment of risk of bias in included studies}

Two review authors (KK and DE) will independently assess risk of bias for each study using the criteria outlined in the Cochrane Handbook for Systematic Reviews of Interventions (Higgins). We will resolve any disagreements by discussion or by involving another author (DA or $\mathrm{AB}$ ). We will assess the risk of bias according to the following domains.
1. Random sequence generation.

2. Allocation concealment.

3. Blinding of participants and personnel.

4. Blinding of outcome assessment.

5. Incomplete outcome data.

6. Selective outcome reporting.

7. Other bias.

We will grade each potential source of bias as high, low or unclear and provide a quote from the study report together with a justification for our judgment in the 'Risk of bias' table. We will summarise the risk of bias judgements across different studies for each of the domains listed. We will consider blinding separately for different key outcomes where necessary (e.g. for unblinded outcome assessment, risk of bias for all-cause mortality may be very different than for a patient reported pain scale). Where information on risk of bias relates to unpublished data or correspondence with a trialist, we will note this in the 'Risk of bias' table.

When considering treatment effects, we will take into account the risk of bias for the studies that contribute to that outcome.

\section{Assesment of bias in conducting the systematic review}

We will conduct the review according to this published protocol and report any deviations in the 'Differences between protocol and review' section of the systematic review.

\section{Measures of treatment effect}

We will analyse dichotomous data as odds ratios and continuous data as mean difference or standardised mean difference. We will enter data presented as a scale with a consistent direction of effect. We will narratively describe skewed data reported as medians and interquartile ranges. We will analyse data from crossover trials using generic inverse variance (GIV) and only if double-counting of participants has been accounted for. If raw data and adjusted analyses (e.g. accounting for baseline differences) are both presented, we will use the latter.

We will undertake meta-analyses only where meaningful i.e. if the treatments, participants and the underlying clinical question are similar enough for pooling to make sense.

Where multiple trial arms are reported in a single trial, we will include only the relevant arms. If two comparisons (e.g. drug A versus placebo and drug $B$ versus placebo) are combined in the same meta-analysis, we will halve the control group to avoid double-counting.

If change from baseline and endpoint scores are available for continuous data, we will use change from baseline unless the majority of studies report endpoint scores. If a study reports outcomes at multiple time-points, we will use the end-of-study measurement. When an analysis using only participants who completed the trial and an analysis which imputes data for participants who were ran- 
domised but did not provide endpoint data (e.g. last observation carried forward) are both available, we will use the latter.

For dichotomous outcomes, we will assume equivalence of treatments if the odds ratio estimate and its $95 \%$ confidence intervals are between the pre-defined arbitrary limits of 0.9 and 1.1 .

\section{Unit of analysis issues}

For dichotomous outcomes, we will use participants rather than events as the unit of analysis (i.e. number of adults admitted to hospital rather than number of admissions per adult). However, if exacerbations are reported as rate ratios we will analyse them on this basis.

\section{Dealing with missing data}

We will contact investigators or study sponsors in order to verify key study characteristics and obtain missing numerical outcome data where possible (e.g. when a study is identified as abstract only). Where this is not possible, and the missing data are thought to introduce serious bias, we will explore the impact of including such studies in the overall assessment of results using a sensitivity analysis.

\section{Assessment of heterogeneity}

We will use the $\mathrm{I}^{2}$ statistic to measure heterogeneity among the trials in each analysis. If we identify substantial heterogeneity (e.g. $\mathrm{I}^{2}$ greater than $30 \%$ ) we will report it and explore possible causes by prespecified subgroup analysis.

\section{Assessment of reporting biases}

If we are able to pool more than 10 trials, we will create and examine a funnel plot to explore possible small study and publication biases.

\section{Data synthesis}

We will use a random-effects model for all analyses as we expect variation in effects due to differences in study populations and methods. We will perform sensitivity analyses with fixed-effects.

\section{Summary of findings table}

We will create a 'Summary of findings' table to present results for all of the named outcomes. We will use the five GRADE considerations (study limitations, consistency of effect, imprecision, indirectness and publication bias) to assess the quality of a body of evidence as it relates to the studies which contribute data to the meta-analyses for the prespecified outcomes. We will apply methods and recommendations described in Section 8.5 and Chapter 12 of the Cochrane Handbook for Systematic Reviews of Interventions (Higgins) using GRADEpro software (Brozek 2008). We will justify all decisions to down- or up-grade the quality of studies using footnotes and we will make comments to aid reader's understanding of the review where necessary.

\section{Subgroup analysis and investigation of heterogeneity}

We plan to carry out the following subgroup analyses for the primary outcomes:

1. Duration of therapy ( $\leq 6$ months, $>6$ months)

2. Corticosteroid dose in the control group (according to GINA 2014-defined low, medium and high cut-offs)

3. Dose and type of LAMA (e.g. tiotropium handihaler 18 $\mathrm{mcg}$, tiotropium respimat $5 \mathrm{mcg}$ )

We will use the formal test for subgroup interactions in Review Manager (RevMan).

\section{Sensitivity analysis}

We plan to carry out sensitivity analyses on the primary outcomes, excluding the following:

1. Unpublished data

2. Studies at high risk of bias for blinding of participants and personnel

3. Crossover studies*

*There may be longer term effects of ICS which do not wash out before a subsequent treatment is started in crossover trials, especially at higher doses.

\section{A C K NOW LEDGEMENTS}

We thank Elizabeth Stovold for designing and running the searches.

Chris Cates was the Editor for this review and commented critically on the review.

The background and methods section of this protocol/review is based on a standard template used by Cochrane Airways Group.

CRG Funding Acknowledgement: The National Institute for Health Research (NIHR) is the largest single funder of the Cochrane Airways Group.

Disclaimer: The views and opinions expressed therein are those of the review authors and do not necessarily reflect those of the NIHR, the NHS or the Department of Health. 


\section{R E F E R E N C E S}

\section{Additional references}

Adams 2008

Adams NP, Bestall JB, Malouf R, Lasserson TJ, Jones PW. Beclomethasone versus placebo for chronic asthma. Cochrane Database of Systematic Reviews 2008, Issue 4. [DOI: 10.1002/14651858.CD002738.pub2]

Adams 2008a

Adams NP, Bestall JC, Lasserson TJ, Jones P, Cates CJ. Fluticasone versus placebo for chronic asthma in adults and children. Cochrane Database of Systematic Reviews 2008, Issue 4. [DOI: 10.1002/14651858.CD003135.pub4]

Bateman 2004

Bateman ED, Boushey HA, Bousquet J, Busse WW, Clark TJ, Pauwels RA, et al.Can guideline-defined asthma control be achieved? The gaining optimal asthma control study. American Journal of Respiratory and Critical Care Medicine 170;8:836-44.

BNF

Joint Formulary Committee. British National Formulary (online). http://www.medicinescomplete.com. London: BMJ Group and Pharmaceutical Press, accessed 27 August 2014.

Brozek 2008

Brozek J, Oxman A, Schünemann H. GradePro Version 3.2 for Windows. McMaster University, 2014, 2008.

Chauhan 2014

Chauhan BF, Ducharme FM. Addition to inhaled corticosteroids of long-acting beta2-agonists versus antileukotrienes for chronic asthma. Cochrane Database of Systematic Reviews 2014, Issue 1. [DOI: 10.1002/ 14651858.CD003137.pub5]

\section{Chong 2012}

Chong J, Karner C, Poole P. Tiotropium versus long-acting beta-agonists for stable chronic obstructive pulmonary disease. Cochrane Database of Systematic Reviews 2012, Issue 9. [DOI: 10.1002/14651858.CD009157.pub2]

Ducharme 2008

Ducharme FM, Ni Chroinin M, Greenstone I, Lasserson TJ. Addition of long-acting beta2-agonists to inhaled corticosteroids versus same dose inhaled corticosteroids for chronic asthma in adults and children. Cochrane Database of Systematic Reviews 2008, Issue 5. [DOI: 10.1002/ 14651858.CD005535.pub2]

Ducharme 2010

Ducharme FM, Ni Choinin M, Greenstone I, Lasserson TJ. Addition of long-acting beta2-agonists to inhaled steroids versus higher dose inhaled steroids in adults and children with persistent asthma. Cochrane Database of Systematic Reviews 2010, Issue 4. [DOI: 10.1002/ 14651858.CD005533.pub2]

eMC 2014

License extension 19th September 2014: Spiriva Respimat 2.5 micrograms solution for inhalation. https:/ /www.medicines.org.uk/emc/history/20134 (accessed 24

October 2014).

GINA 2014

Global Initiative for Asthma. Global Strategy For Asthma Management And Prevention. http://www.ginasthma.org/ (accessed 22nd August 2014).

Global Asthma Report 2011

International Union Against Tuberculosis and Lung Disease. The Global Asthma Report 2011. http://theunion.org/ what-we-do/publications/technical/global-asthma-report (accessed 28th August 2014).

\section{Gosens 2006}

Gosens R, Zaagsma J, Meurs H, Halayko AJ. Muscarinic receptor signaling in the pathophysiology of asthma and COPD. Respiratory Research 2006;7(1):73.

\section{Higgins}

Higgins JPT, Green S (editors). Cochrane Handbook for Systematic Reviews of Interventions Version 5.1 [updated March 2011]. The Cochrane Collaboration, 2011. www.cochrane-handbook.org.

\section{Karner 2014}

Karner C, Chong J, Poole P. Tiotropium versus placebo for chronic obstructive pulmonary disease. Cochrane Database of Systematic Reviews 2014, Issue 7. [DOI: 10.1002/ 14651858.CD009285.pub3]

\section{Lipworth 2014}

Lipworth BJ. Emerging role of long acting muscarinic antagonists for asthma. British Journal of Clinical Pharmacology 2014;77(1):55-62.

\section{Moulton 2011}

Moulton BC, Fryer AD. Muscarinic receptor antagonists, from folklore to pharmacology; finding drugs that actually work in asthma and COPD. British Journal of Clinical Pharmacology 2011;163(1):44-52.

Ni 2014

Ni H, Soe Z, Moe S. Aclidinium bromide for stable chronic obstructive pulmonary disease. Cochrane Database of Systematic Reviews 2014, Issue 9. [DOI: 10.1002/ 14651858.CD010509.pub2]

NICE 2013

National Institute for Health and Care Excellence. NICE advice [KTT5]High-dose inhaled corticosteroids in asthma http://www.nice.org.uk/Advice/ktt5 (accessed 29th August 2014).

\section{NRAD 2014}

Royal College of Physicians. Why asthma still kills: the national review of asthma deaths. https:// www.rcplondon.ac.uk/sites/default/files/why-asthma-stillkills-full-report.pdf (accessed 28th August 2014).

Review Manager (RevMan)

The Nordic Cochrane Centre, The Cochrane Collaboration. Review Manager (RevMan). 5.3. Copenhagen: The Nordic Cochrane Centre, The Cochrane Collaboration, 2014. 
Tee 2009

Tee A, Koh MS, Gibson PG, Lasserson TJ, Wilson A, Irving LB. Long-acting beta2-agonists versus theophylline for maintenance treatment of asthma. Cochrane Database of Systematic Reviews 2009, Issue 3. [DOI: 10.1002/ 14651858.CD001281.pub2]

Verhamme 2013

Verhamme KM, Afonso A, Romio S, Stricker BC, Brusselle GG, Sturkenboom MC. Use of tiotropium Respimat Soft Mist Inhaler versus HandiHaler and mortality in patient with COPD. European Respiratory Journal 42;3:606-15.

Wise 2013

Wise RA, Anzueto A, Cotton D, Dahl R, Devins T, Disse $\mathrm{B}$, et al.Tiotropium Respimat Inhaler and the risk of death in COPD. New England Journal of Medicine 2013;369: 1491-1501.

* Indicates the major publication for the study

\section{A P P E N D I C E S}

\section{Appendix I. Sources and search methods for the Cochrane Airways Group Specialised Register (CAGR)}

Electronic searches: core databases

\begin{tabular}{ll}
\hline Database & Frequency of search \\
\hline CENTRAL (the Cochrane Library) & Monthly \\
\hline MEDLINE (Ovid) & Weekly \\
\hline Embase (Ovid) & Weekly \\
\hline PsycINFO (Ovid) & Monthly \\
\hline CINAHL (EBSCO) & Monthly \\
\hline AMED (EBSCO) & Monthly \\
\hline
\end{tabular}

Hand-searches: core respiratory conference abstracts 


\begin{tabular}{l|l}
\hline Conference & Years searched \\
\hline American Academy of Allergy, Asthma and Immunology (AAAAI) & 2001 onwards \\
\hline American Thoracic Society (ATS) & 2001 onwards \\
\hline Asia Pacific Society of Respirology (APSR) & 2004 onwards \\
\hline British Thoracic Society Winter Meeting (BTS) & 2000 onwards \\
\hline Chest Meeting & 2003 onwards \\
\hline European Respiratory Society (ERS) & $1992,1994,2000$ onwards \\
\hline International Primary Care Respiratory Group Congress (IPCRG) & 2002 onwards \\
\hline Thoracic Society of Australia and New Zealand (TSANZ) & 1999 onwards \\
\hline
\end{tabular}

\section{MEDLINE search strategy used to identify trials for the CAGR}

\section{Asthma search}

1. exp Asthma/

2. asthma\$.mp.

3. (antiasthma $\$$ or anti-asthma $\$$ ).mp.

4. Respiratory Sounds/

5. wheez\$.mp.

6. Bronchial Spasm/

7. bronchospas $\$ . m p$.

8. (bronch $\$$ adj3 spasm\$).mp.

9. bronchoconstrict $\$ . \mathrm{mp}$.

10. exp Bronchoconstriction/

11. (bronch\$ adj3 constrict\$).mp.

12. Bronchial Hyperreactivity/

13. Respiratory Hypersensitivity/

14. ((bronchial\$ or respiratory or airway $\$$ or lung $\$$ ) adj3 (hypersensitiv\$ or hyperreactiv\$ or allerg $\$$ or insufficiency)).mp.

15. ((dust or mite\$) adj3 (allerg\$ or hypersensitiv\$)).mp.

16. or/1-15

\section{Filter to identify RCTs}

1. exp "clinical trial [publication type]"/

2. (randomised or randomised).ab,ti.

3. placebo.ab,ti.

4. dt.fs.

5. randomly.ab,ti.

6. trial.ab,ti.

7. groups.ab,ti.

Long-acting muscarinic antagonists (LAMA) added to inhaled corticosteroids (ICS) versus higher dose ICS for adults with asthma

\section{(Protocol)}

Copyright $\Subset 2014$ The Cochrane Collaboration. Published by John Wiley \& Sons, Ltd. 
8. or/1-7

9. Animals/

10. Humans/

11. 9 not (9 and 10)

12. 8 not 11

The MEDLINE strategy and RCT filter are adapted to identify trials in other electronic databases.

\section{Appendix 2. Search strategy to identify relevant trials from the CAGR}

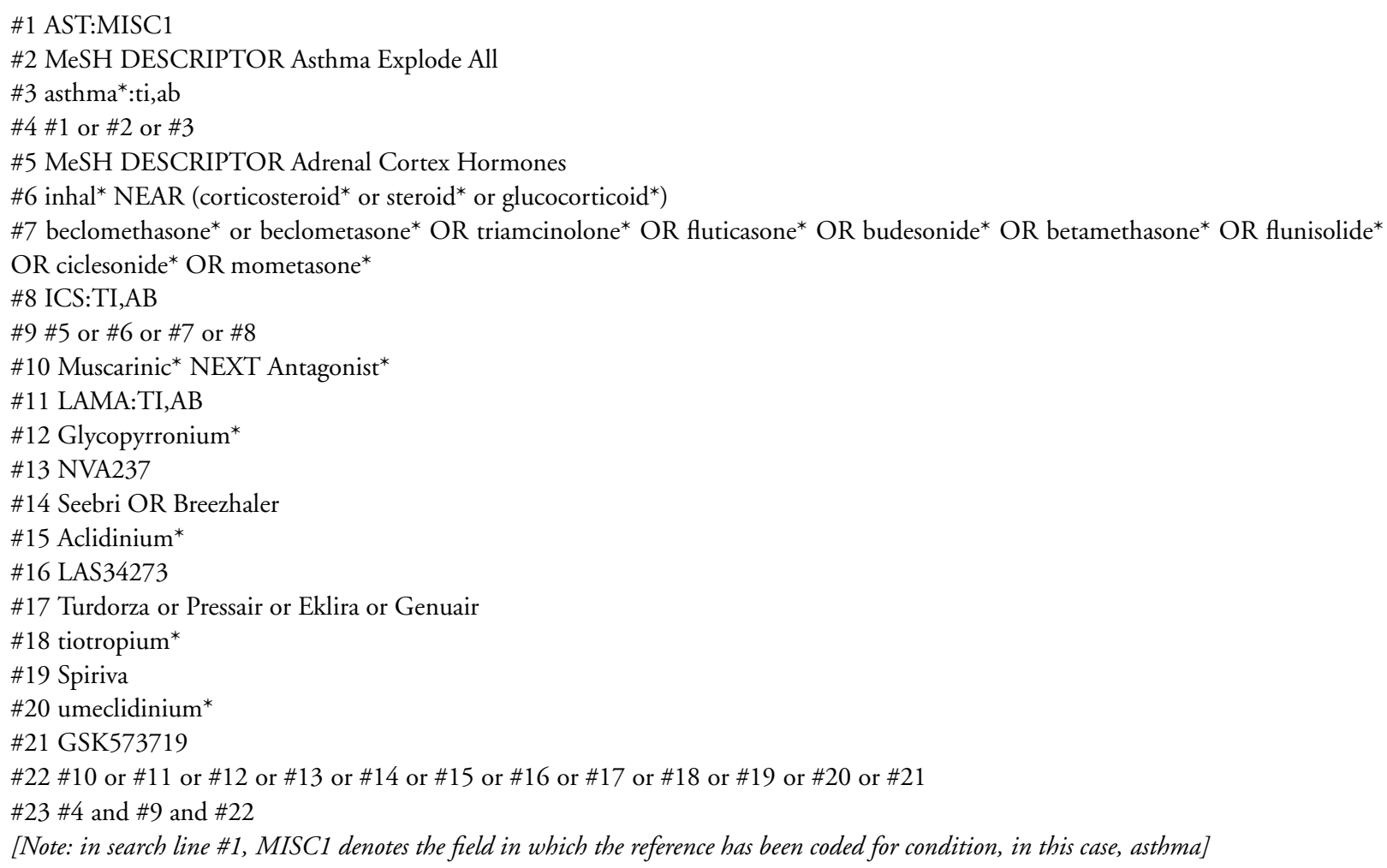

\section{CONTRIBUTIONSOFAUTHORS}

Kayleigh Kew wrote the background and methods, with edits and critical input from Debbie Allison, Anne Boyter and David Evans. All authors approved the final version of the document.

\section{DECLARATIONSOF INTEREST}

Kayleigh Kew: none known

Debbie Allison: none known

Anne Boyter: none known

David Evans: none known 


\section{SOURCES OF SUPPORT}

\section{Internal sources}

- No sources of support supplied

\section{External sources}

- National Intitute of Health Research, UK.

Evidence to guide care in adults and children with asthma, 13/89/14 